\title{
Pilot Tone based CD and PMD Monitoring Technique for Photonic Networks
}

\author{
Koushik Barman* and Mritunjay Kumar Rai \\ School of Electronic and Electrical Engineering, Lovely Professional University, Phagwar - 144411,Punjab,India; \\ koushik.15737@lpu.co.in
}

\begin{abstract}
Objectives: This paper aims at evaluation of pilot tone based Chromatic dispersion and Polarization mode dispersion monitoring technique and to find out a suitable solution for simultaneous monitoring of CD and PMD in dynamic optical network. Method: A pilot tone of $2 \mathrm{GHz}$ to $8 \mathrm{GHz}$ has been send to each channel of a WDM Link for the monitoring of performance degradation of composite signal during the transmission. CD and PMD causes phase shift of LSB and USB of the amplitude modulated pilot tone which results variation of amplitude of the pilot tone. The amplitude of the pilot tome is the parameter by which measurement of different quantities such as optical power, wavelength, OSNR, CD and PMD can be done in a dynamic optical network. Findings: From the various literatures it has been observed that Optical network is switching from static form to dynamic form. Implementation of plug and play devices, adaptive modulation techniques and various dynamic services introduce complexity in photonic network. Due to highly dynamic and heterogeneous nature of optical network, investigation and implementation of appropriate performance monitoring technique is a major challenge for the researchers. This paper presents a pilot tone based method for simultaneous monitoring of CD and PMD. The proposed method is suitable for dynamic optical network and the simulation results shows low frequency pilot tones provides better resolution for both $\mathrm{CD}$ and PMD monitoring technique. The simulation result shows that $2 \mathrm{GHz}$ pilot tone provides CD measurement range upto $16000 \mathrm{ps} / \mathrm{nm}$ and PMD DGD value upto 50ps for the tone power variation of -10 to 0dB. Applications: The proposed techniques can be used for simultaneous CD and PMD monitoring of dynamic WDM optical networks.
\end{abstract}

Keywords: Bit Error Rate, Chromatic Dispersion, Optical Impairments, Polarization Mode Dispersion, Wavelength Division Multiplexing

\section{Introduction}

Optical networks are switching towards high capacity non static transparent self-managed WDM mesh networks. In future there will be an option to simply 'plug \& play' an optical node into an existing network in such a way that the network itself can allocate resources to ensure error free transmission. The channel capacity of present optical network is more than $10 \mathrm{~Tb} / \mathrm{s}$. Therefor a small disturbance in such network creates huge problem to the society. Naturally to assure and enable robust and cost-effective automated operation with higher stability, configurability and flexibility in term of the required QoS, the future optical network should probably be able to intelligently monitor the physical state of the network as well as the quality of propagating data signals, automatically diagnose and repair the network, allocate resources and redirect traffic ${ }^{1,2}$. Figure 1 shows various characteristics of heterogeneous photonic networks which includes variable bitrates, circuit plus packet switching, multiple wavelength ranges, variable QoS, subcarrier multiplexing, adaptive modulation format etc. Therefore it is also require to investigate suitable optical performance monitoring technique for optical impairments so that error free high quality transmission is possible through future optical network.

Chromatic dispersion (CD) and polarization mode dispersion (PMD) are two unavoidable impairments of

*Author for correspondence 
high bit rate and long distance optical communication network ${ }^{5}$. Their degradation impact on optical network increases as the nature of the network switches from static to dynamic. There are various methods reported for individual monitoring of $\mathrm{CD}$ and PMD for static optical network but a single technique for simultaneous monitoring of $\mathrm{CD}$ and PMD for dynamic heterogeneous network is a research challenge. Recent research is going on for performance monitoring of elastic optical network. OSNR and CD are the two prime parameters for performance monitoring of self-adapting, dynamic elastic optical network $\stackrel{11}{ }$.

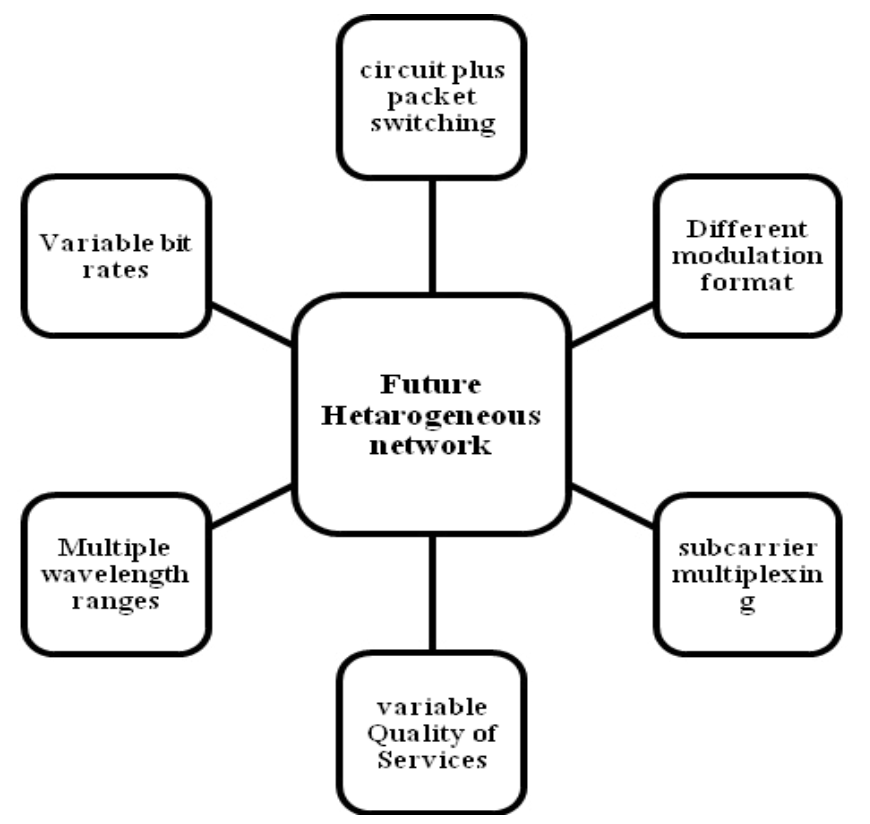

Figure 1. Charecteriscis of future photonc networks.

\section{Various Types of OPM Techniques}

Optical performance monitoring techniques are categorized into Time domain techniques and Frequency domain techniques. Figure 2 illustrates classification of OPM.

\subsection{Time Domain OPM Techniques}

Time domain techniques are basically based on observation of several samples taken at a specific distance of fibre network and then analysing the degradation effect on that sampled signal will provide the overall calculation of degradation effect of whole signal. They are again classified into synchronous and asynchronous techniques ${ }^{\underline{3}}$ based on the way of taking samples form the network. In case of synchronous sampling fixed time $Q$ factor $\left(Q_{t}\right)$ is the measurement parameter for analyzing the samples. On the Other hand average $\mathrm{Q}$ factor $\left(\mathrm{Q}_{\text {avg }}\right)$ is obtained by the help of asynchronous sampling. The BER of the signal is $10^{-10}$ if the measured $Q_{t}$ value is 16.4 for a 10 Gbps signal ${ }^{4}$. As the bit rate increases the value of $Q_{t}$ decreases. In the literature (2), $Q_{t}$ has been calculated by forming the eye diagram. The Eye diagram is obtained by plotting all sampling points in time scale with calculated sampling time period from the following equation.

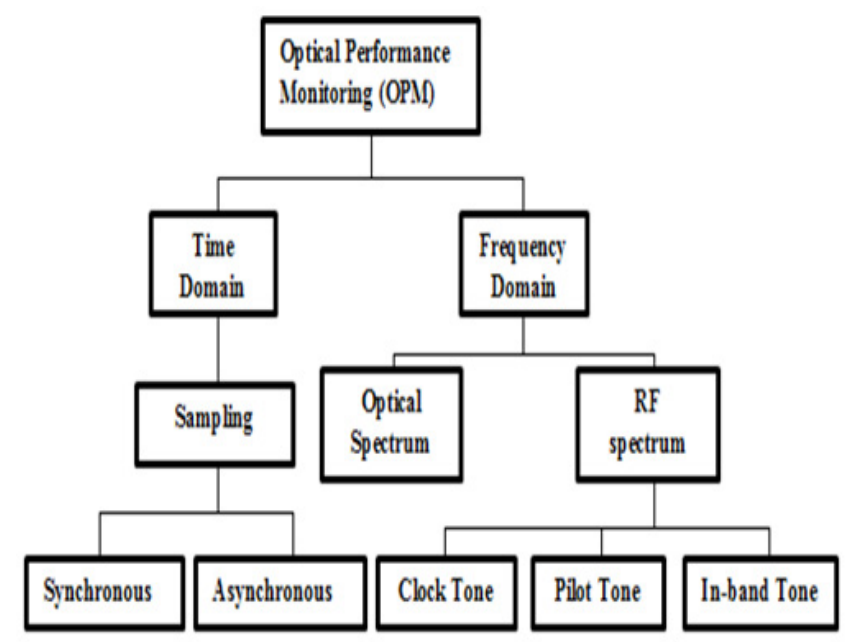

Figure 2. Different Types of OPM techniques.

$$
\mathrm{T}_{\text {step }}=\frac{1}{f_{c}}-\frac{1}{m f_{s}}=\frac{1}{k f_{s}}
$$

Where $\mathrm{f}_{\mathrm{s}}$ is signal bit rate, $\mathrm{f}_{\mathrm{c}}$ is sampling clock rate, $T_{\text {step }}$ is sampling time interval, $\mathrm{n}, \mathrm{m}$ are natural numbers and $\mathrm{k}$ is the number of sampling points per time slot. $\mathrm{Q}_{\mathrm{t}}$ is given by,

$$
\mathrm{Q}_{\mathrm{t}}=\left|\mu_{1}-\mu_{0}\right| /\left(\sigma_{1}+\sigma_{2}\right)
$$

This method is not appropriate for WDM network. Sampling of each channel of a WDM system then again resembling the sampled signal for each channel makes it complicated and costly system.

\subsection{Frequency Domain OPM Techniques}

Frequency domain techniques are more accurate than time domain technique and they are classified into two category i.e Optical spectrum and RF spectrum.

In case of optical spectrum method carrier frequency and optical noise information can be obtain by analyzing 
the optical spectrum. Optical spectrum analyzers are also broadly available in the market. It build by a tunable band pass filter or a diffraction grating. These types of OPMs have capability to monitor the optical power as well as the wavelength of individual channel of a WDM link. It also estimates the optical signal-to-noise ratio (OSNR) by checking of the signal'sASE level. It is reported in papers that is method is quit erroneous for dynamically reconfigurable WDM network because in this type of network each channel travels different routes and each route has different number of optical amplifiers ${ }^{8,9}$.

On the other hand RF spectrum based OPM techniques are more familiar than optical spectrum methods, as these methods are more accurate, easier and cost effective to implement in dynamic optical networks. The well-known optical modulation formats are non-returnto-zero (NRZ), return-to-zero (RZ), carrier-suppressed return-to-zero (CS-RZ). The beauty of this technique is that it is applicable for all these formats. This technique is also suitable for bit-rates more than $40 \mathrm{Gbit} / \mathrm{s}^{4,5}$. This technique is not intrusive. It also not require to modify transmitter. The monitor response time is very narrow, more specifically in the order of sub microseconds. Therefore real-time monitoring is possible. Also this technique is suitable for dynamic networks.

RF spectrum methods are classified into three category i.e. Clock Tone, Pilot Tone and In-band Tone techniques $\frac{6-8}{}$.

\section{Proposed Pilot Tone based Technique}

\subsection{Basic theories of pilot tone method}

A pilot tone is a low frequency signal also can be modulated using carrier signal. It is added with each channel of WDM transmitter. Therefore, in a WDM system, different tone frequency pilot tone can be added to each channel. This pilot tone can be removed from any node in the optical network. The amplitude of the pilot tome is the parameter by which measurement of different quantities such as optical power, wavelength, OSNR, CD and PMD can be done in a dynamic optical network. The amplitude modulated pilot tone when travels through the optical network path, due to chromatic dispersion it causes phase shift between LSB and USB. This phase shift causes RF power fading at the receiver. The normalized magnitudes of the received AM pilot tone can be obtained by 1

$$
\mathrm{P}_{\mathrm{AM}} \infty \mathrm{m} \cos \left(\frac{\pi D L \lambda^{2} f^{2}}{C}\right)
$$

Where $\mathrm{c}$ is the speed of light, $\lambda$ is the wavelength, and $\mathrm{m}$ and $\mathrm{f}$ are the modulation index and tone frequency respectively. $\mathrm{D}$ and $\mathrm{L}$ are the dispersion parameter and fiber length respectively. Therefore it is clear that dispersion depends on the received power of pilot tone. So by extracting the pilot tone at any node and measuring its amplitude level, calculation of corresponding dispersion for a given length of fiber can be obtained. The resolution ${ }^{1}$ is define as the ratio of power obtained and multiplication of dispersion and fiber length, i.e $\triangle \mathrm{PAM} / \Delta(\mathrm{DL})$. The maximum measurement range is given by $\mathrm{GVD}_{\max }=\mathrm{c} /$ $2 \lambda^{2} \mathrm{f}^{2}$.

As the tone frequency increases the resolution also improves but the measurement range decreases. For 4 $\mathrm{GHz}$ pilot tone resolution is $4000 \mathrm{ps} / \mathrm{nm}$. For $8 \mathrm{GHz}$ pilot tone, the average resolution and measurement range are given by $0.006 \% \mathrm{ps} / \mathrm{nm}$ and $1000 \mathrm{ps} / \mathrm{nm}$, respectively ${ }^{1}$. Therefore if the tone frequency is $2 \mathrm{GHz}$, maximum measurement range achieved more than $15000 \mathrm{ps} / \mathrm{nm}$.

There are several limitation of pilot tone based monitoring technique. The modulated pilot tone (AM or PM) is sensitive to the effect of PMD. The effect of PMD on the received RF power can be obtained by the following equation.

$$
\mathrm{P}_{\infty} 1-4 \gamma(1-\gamma) \sin ^{2}(\pi \mathrm{f} \Delta \tau)
$$

Where $\Delta \tau$ and $\gamma$ are the differential group delay (DGD) and the power ratio between the fast and slow axes, respectively. Equation 4 shows that the power level of pilot tone decreases drastically as the group velocity dispersion occurs or effect of PMD impacts the CD monitoring range for the same pilot tone. By using a Broadband Light Source, the effect of PMD on CD monitoring can be controlled. If the pilot tone is generated by the Broadband Light source then the RF power variation of the received AM pilot tone, effected by $\mathrm{CD}$, can be obtained by the following equation $\frac{4,10}{10}$

$$
\mathrm{P} \infty \exp \left(-\left(\pi / 2 \mathrm{DL} \sigma_{\lambda} \mathrm{f}\right)^{2}\right)
$$

Where $\sigma_{\lambda}$ is the spectral width of the BLS, D is the dispersion parameter, $\mathrm{L}$ is the fiber length, and $\mathrm{f}$ is the tone frequency. From the above relation it is clear that a $2 \mathrm{GHz}$ 
pilot tone is needed to monitor Chromatic dispersion upto $1000 \mathrm{ps} / \mathrm{nm}$ (assuming that $\sigma_{\lambda}=0.8 \mathrm{~nm}$ ), therefore the performance is less sensitive to PMD.

\subsection{Proposed Method and Simulation Result}

The concept is to add a RF signal (pilot tone) of frequency $2 \mathrm{GHz}$ to $10 \mathrm{GHz}$ with modulated signal using an optical intensity modulator such as a Lithium Niobate (LiNbO3) Mach-Zehnder, at the transmitter. After transmission the $\mathrm{RF}$ tone power is extracted in the monitoring unit. Amplitude of the received RF power can be a measure of CD.

Chromatic dispersion and PMD depends on power fading received by the pilot tone. This power fading may be used as measurement parameter for monitoring of $C D$ and PMD.

Pilot tones of range 2 to $10 \mathrm{GHz}$ have been used for simulating the proposed technique. Figure 3 shows the simulation result of $\mathrm{CD}$ measurement whereas Figure 4 shows the PMD measurement result of same range of pilot tone frequencies.

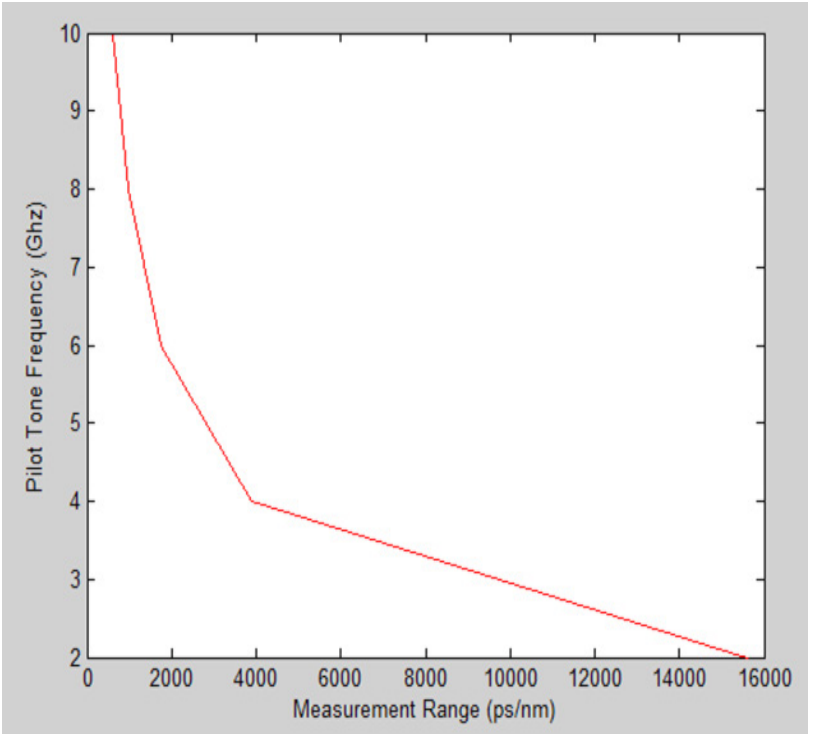

Figure 3. CD measurement range for $2 \mathrm{GHz}$ to $10 \mathrm{GHz}$ pilot tone.

\section{Conclusion}

Simultaneous monitoring of CD and PMD is very difficult for highly dynamic optical network. The proposed technique provides higher resolution and can be applied for $>40$ Gbps networks. Pilot tone based monitoring technique is cost effective and easy to implement. The pro- posed technique deals with integration of multiple OPM techniques i.e both $\mathrm{CD}$ and PMD which will reduce the overall cost. The simulation result shows that $2 \mathrm{GHz}$ pilot tone provides CD measurement range upto $16000 \mathrm{ps} / \mathrm{nm}$ and PMD DGD value upto 50ps for the tone power variation of -10 to $0 \mathrm{~dB}$. The proposed technique has higher resolution and measurement range.

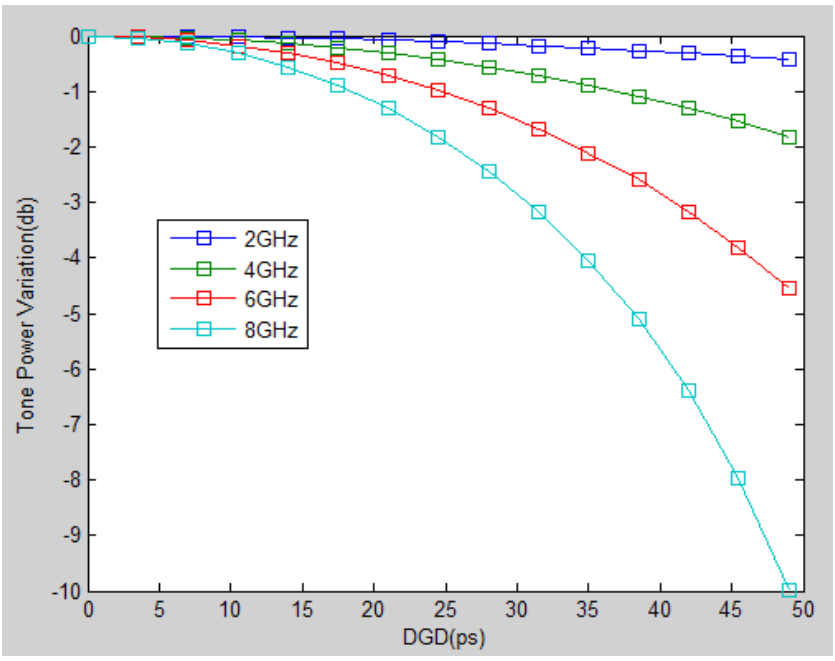

Figure 4. Measurement of Polarization mode dispersion on received $\mathrm{RF}$ tone power $0 \mathrm{~dB}$ to $-10 \mathrm{~dB}$.

\section{References}

1. Park KJ, et al. Performance comparisons of chromatic dispersion-monitoring techniques using pilot tones ,IEEE Photonic Technology. 2003 June; 15 (6).

2. Shak I, Hidehiko Takara, Satoki Kawanishi. Simple Q Factor Monitoring for BER Estimation Using Opened Eye Diagrams Captured by High-Speed Asynchronous Electrooptical Sampling IEEE Photonic Technology. 2003 April; 15(4).

3. Changyuan Yu, Yi Yu. Optical performance monitoring in fiber transmission systems based on electrical sampling technique IEEE ICTON. 2014; 1-4.

4. Choi HY, Paul K, Park J, Chung YC. Chromatic Dispersion Monitoring Technique Using Pilot Tone Carried by broadband light source IEEE. 2009; 21(9).

5. Kilperet DC al. Optical performance monitoring, J. Lightw. Technology. 2004 Jan; (22):294-304.

6. Park PKJ, Jun SB, Chung YC. Chromatic dispersion monitoring technique based on chirped pilot tones, Opt. Communication. 2006 Oct; (266): 280-83.

7. Li Z, Li G. In-line performance monitoring for RZ-DPSK signals using asynchronous amplitude histogram evaluation, IEEE Photonic Technol. Lett., 2006;(18): 472-74. 
8. Lamia Baker-Mefla, Benn C. Thomsen, John E.Mitchell, PolinaBayvel. Multi-Impairment WDM Optical Performance Monitoring for Burst Switched Networks JLT, 2010 Dec: 28(23).

9. Trevor B. Anderson, Adam Kowalczyk, KeClarke, Sarah D. Dods Don Hewitt, Jonathan. Multi Impairment Monitoring for Optical Networks JLT, 2009 Aug: 27 (16).
10. Barman K, Kassa S, Kosale D. Advance Performance Monitoring Techniques for Future Photonic Network, $5^{\text {th }}$ national conference, India Com 2011.

11. Calvin CK Chan, Kam-Hon Tse, Tianwai Bo, Shuang Gao. Optical performance monitoring in elastic optical OFDM networks, IEEE conference Photonics in Switching (PS) 2015.p.145-47 